\title{
A UNIQUENESS THEOREM FOR THE REDUCED WAVE EQUATION ${ }^{1}$
}

\author{
JULIUS KANE
}

1. Introduction. Mixed boundary conditions of the form

$$
\frac{\partial u}{\partial y}+\lambda u=0
$$

have been used with success in electromagnetic theory to describe materials of high, but imperfect conductivity $[1 ; 2 ; 3]$. The parameter $\lambda$ can be chosen to accurately reproduce the phenomenology at the conductor's surface; other choices of $\lambda$ lead to a boundary condition which can describe reactive surfaces that support slow waves or surface waves $[4 ; 5 ; 6]$.

The utility of the boundary condition (1) to model the physics at complex interfaces has led to an investigation [7] of another boundary condition with more parameters

$$
\frac{1}{i} \frac{\partial u}{\partial y}+A u+B \frac{\partial^{2} u}{\partial x^{2}}=0, \quad i=+(-1)^{1 / 2},
$$

which can be chosen to approximate more involved situations, e.g. diffraction at a dielectric-dielectric interface. The physical justification for this procedure is presented elsewhere [7]. Here we seek to show that this is a well-posed approach by proving the uniqueness of the solution of the standard problem of a radiating line source above a plane boundary at which a condition of the form (2) is imposed. The proof of uniqueness is nonstandard owing to the presence of the second derivative in the boundary condition (2).

2. Statement of the problem. We seek to show that there exists one and only one solution of the reduced wave equation

$$
\left(\nabla^{2}+k^{2}\right) u=\delta(x) \delta(y-h), \quad y \geqq 0,
$$

with a source at $(0, h)$, which satisfies the boundary condition (2)

Presented to the Society, April 23, 1960 under the title $A$ uniqueness theorem for the wave equation under a class of mixed boundary conditions; received by the editors July 13, 1960.

1 The research in this report was sponsored in part by the Electronics Research Directorate of the Air Force Cambridge Research Center, Air Research and Development Command, under Contract No. AF 19(604)-5238, and in part by the American Petroleum Institute. 
at $y=0$. For simplicity, we shall confine our discussion to the case where $k$ in (3), and $A$ and $B$ in (2) are real. It is customary to specify the radiation condition at infinity

$$
\lim _{\rho \rightarrow \infty} \rho^{1 / 2}\left[\frac{\partial u}{\partial \rho}-i k u\right]=0, \quad 0 \leqq \theta \leqq \pi,
$$

which is written in terms of the polar coordinates

$$
\left\{\begin{array}{l}
\rho^{2}=x^{2}+y^{2} \\
\theta=\arctan \frac{y}{x} .
\end{array}\right.
$$

However, for our proof, we shall require a more explicit statement at infinity.

3. Existence. A solution of (2), (3), and (4) which has the following behavior at infinity

$$
\lim _{\rho \rightarrow \infty} u(\rho, \theta)=p(\theta) \frac{e^{i k \rho}}{(k \rho)^{1 / 2}}+O\left[(k \rho)^{-8 / 2}\right], \quad 0 \leqq \theta \leqq \pi,
$$

can be obtained [7] by Fourier transforms, where $p(\theta)$, the far-field amplitude or pattern function, is an analytic function of $\theta$ explicitly determined by $A, B, h$ and $k$.

4. Uniqueness. We utilize the far-field behavior (6), rather than the usual Sommerfeld radiation condition $(4)$, of $u(x, y)$ to prove the uniqueness theorem:

THEOREM. Let $u(x, y)$ be any regular function which satisfies (2) and (3) with $A$ and $B$ as arbitrary real constants. If $u(x, y)$ can be developed as

$$
u(\rho, \theta)=g(\theta) \frac{e^{i k \rho}}{(k \rho)^{1 / 2}}+O\left[(k \rho)^{-3 / 2}\right], \quad 0 \leqq \theta \leqq \pi,
$$

for $k \rho \gg 1$, where $g(\theta)$ is any pattern function, then $u(x, y)$ is unique.

PROOF. Let $u(x, y)=u_{1}(x, y)-u_{2}(x, y)$ where $u_{1}$ and $u_{2}$ are any two functions that satisfy (2), (3), and (7). Then $u(x, y)$ is a solution of the homogeneous wave equation

$$
\left(\nabla^{2}+k^{2}\right) u(x, y)=0
$$

and in addition satisfies (3) and (7). Construct the auxiliary function $v(x, y)$ in terms of $u(x, y)$ and its derivatives as 


$$
v(x, y)=\frac{1}{i} \frac{\partial u}{\partial y}+A u+B \frac{\partial^{2} u}{\partial x^{2}}
$$

then $v(x, y)$ satisfies Dirichlet conditions at $y=0$ as well as the homogeneous wave equation for $y \geqq 0$; we shall first show that $v$ must vanish identically for $y \geqq 0$. This can be seen by an application of Green's theorem about a semi-circle $\Gamma$ of radius $\rho$, to $v(x, y)$ and its conjugate $v^{*}(x, y)$.

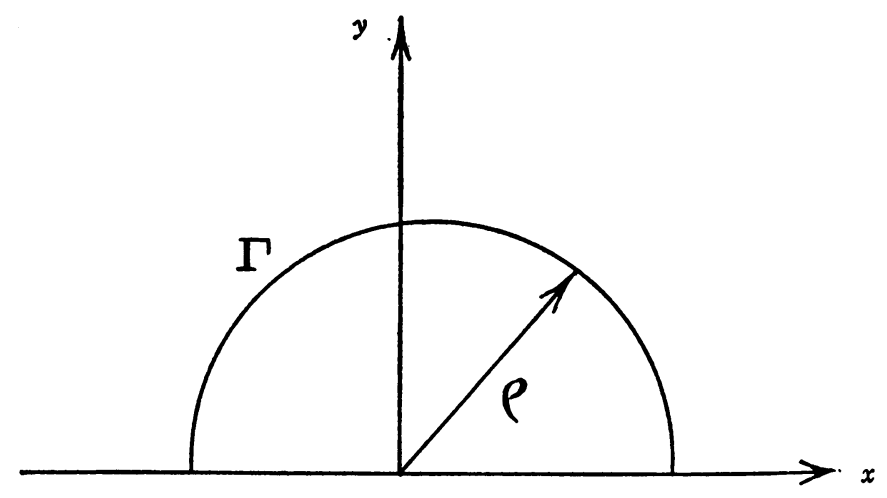

Namely, if $A(\Gamma)$ is the area bounded by $\Gamma$, we have

$$
\int_{\Gamma}\left(v^{*} \frac{\partial v}{\partial n}-v \frac{\partial v^{*}}{\partial n}\right) d s=\int_{A(\Gamma)}\left(v^{*} \nabla^{2} v-v \nabla^{2} v^{*}\right) d \tau,
$$

and by virtue of the boundary condition (2), which implies that $v^{*}(x, 0)=v(x, 0)=0$, it follows that

$$
\int_{0}^{\pi}\left(v^{*} \frac{\partial v}{\partial \rho}-v \frac{\partial v^{*}}{\partial \rho}\right) \rho d \theta=\int_{A(\Gamma)}\left(v^{*} \nabla^{2} v-v \nabla^{2} v^{*}\right) d \tau
$$

Since $k^{2}$ is real, the integral over $A(\Gamma)$ vanishes identically which leaves us with

$$
\int_{0}^{\pi}\left(v^{*} \frac{\partial v}{\partial \rho}-v \frac{\partial v^{*}}{\partial \rho}\right) \rho d \theta=0 .
$$

Let $\rho \rightarrow \infty$, and use the condition (7) to change (10) to the form

$$
\int_{0}^{\pi} q(\theta) q^{*}(\theta) d \theta=0
$$


where $q(\theta)$ is the pattern function for $v(x, y)$; this in turn implies $q(\theta)=0$ a.e., $0 \leqq \theta \leqq \pi$. The function $v(x, y)$ therefore has a vanishing far-field amplitude in this region of definition $y \geqq 0$. We wish to apply a theorem of Rellich [8] in order to show $v \equiv 0$. The relevant theorem applies to a function regular outside some circle. We therefore proceed to extend $v$ as follows: Since $v(x, y)$ vanishes on the $x$-axis it can be analytically continued into the lower half-plane by the reflection principle. Hence $v$ is now regular in the complete $x, y$-plane. We are now in a position to apply Rellich's Theorem. This theorem states that zero is the only wave function which is regular in the entire $x, y$-plane, and which vanishes more rapidly than $O\left(1 / p^{1 / 2}\right)$ for $\rho \rightarrow \infty$ and $\theta \in I$ where $I$ is any interval [8]. Equation (11) therefore implies that $v \equiv 0$.

These results show that any solution $u(x, y)$ of the homogeneous wave equation with the boundary condition (2) satisfies the differential equation

$$
\frac{1}{i} \frac{\partial u}{\partial y}+A u+B \frac{\partial^{2} u}{\partial x^{2}}=0
$$

for all $y \geqq 0$. It is a simple matter to show that this is inconsistent with the assumed asymptotic development (7) thus yielding the desired contradiction. To obtain this result, substitute (7) into (12) and obtain

$$
g(\theta)\left[k \sin \theta+A-B k^{2} \cos ^{2} \theta\right] \frac{e^{i k \rho}}{(k \rho)^{1 / 2}}=O\left[(k \rho)^{-3 / 2}\right], \quad 0 \leqq \theta \leqq \pi .
$$

Equation (13) can be satisfied only if $g(\theta)$ vanishes, or else, if

$$
k \sin \theta+A-B k^{2} \cos ^{2} \theta=0, \quad 0 \leqq \theta \leqq \pi .
$$

Since (14) is a quadratic in $\sin \theta$ it follows that the equation (14) can be satisfied for at most two values of $\sin \theta$. Hence

$$
g(\theta)=0, \quad 0 \leqq \theta \leqq \pi .
$$

A vanishing far-field amplitude (15) is not sufficient to imply that $u(x, y) \equiv 0$. We shall complete the proof by an appeal to Stokes' theorem. Since $u(x, y)$ is a wave function, the integral

$$
\int_{A(\Gamma)}\left(u \nabla^{2} u^{*}-u^{*} \nabla^{2} u\right) d \tau=0
$$

vanishes for any region bounded by a closed curve $\Gamma$ in the upper halfplane. In particular, if $\Gamma$ is a semicircle of radius $\rho$ we can compute 
the integral (16) in another way. If the Laplacian operates on any wave function satisfying (12) we obtain

$$
\nabla^{2} u=-k^{2} u=\frac{1}{i A} \frac{\partial u}{\partial y}+\frac{B}{A} \frac{\partial^{2} u}{\partial x^{2}},
$$

so that (16) becomes

$$
\begin{aligned}
0 & =\int_{A(\Gamma)}\left[-\frac{1}{i A}\left(u u_{y}^{*}+u^{*} u_{y}\right)+\frac{B}{A}\left(u u_{x x}^{*}-u_{x x} u^{*}\right)\right] d \tau \\
& =\int_{A(\Gamma)}\left[-\frac{1}{i A} \frac{\partial}{\partial y}\left(u u^{*}\right) \frac{B}{A} \frac{\partial}{\partial x}\left(u u_{x}^{*}-u_{x} u^{*}\right)\right] d \tau
\end{aligned}
$$

whence, by Stokes' theorem

$$
0=\int_{\Gamma} \frac{B}{A}\left(u u_{x}^{*}-u_{x} u^{*}\right) d y+\frac{1}{i A} u u^{*} d x,
$$

or

$$
0=I_{1}+I_{2}+I_{3}
$$

where

$$
\begin{aligned}
& I_{1}=\frac{B}{A} \int_{0}^{\pi}\left(u u_{x}^{*}-u_{x} u^{*}\right) \rho \cos \theta d \theta, \\
& I_{2}=-\frac{1}{i A} \int_{0}^{\pi} u u^{*} \rho \sin \theta d \theta, \\
& I_{3}=\frac{1}{i A} \int_{-\rho}^{\rho} u u^{*} d x .
\end{aligned}
$$

As $\rho \rightarrow \infty$ the first two integrals $I_{1}$ and $I_{2}$ vanish by (15) since both $u$ and $u_{x}$ behave like $\rho^{-3 / 2}$ as $|\rho| \rightarrow \infty, 0 \leqq \theta \leqq \pi$. We are left with

$$
\int_{-\infty}^{\infty} u u^{*} d x=0
$$

so we conclude that $u$ must vanish on the boundary: $y=0$. Hence $u(x, y) \equiv 0, y \geqq 0$ by the same argument previously applied to the function $v$.

5. Concluding remarks. Similiar analysis will prove uniqueness theorems involving boundary conditions of the form

$$
\frac{1}{i} \frac{\partial u}{\partial y}+\sum_{i=1}^{N} A_{i} \frac{\partial^{2 i} u}{\partial y^{2 i}}=0 \text {. }
$$


That is, it is possible to have available as many parameters as necessary to construct boundary conditions which model diffraction at various interfaces. However, these constants can only be coefficients of even order derivatives or reciprocity will be sacrificed [7].

Acknowledgment. It is a pleasure to acknowledge the helpful discussions and comments of Professor Samuel N. Karp of New York University.

\section{REFERENCES}

1. G. A. Grünberg, Suggestions for a theory of coastal refraction, Phys. Rev. vol. 63 (1943) p. 185.

2. J. Bazer and S. N. Karp, Propagation of plane electromagnetic waves past a shoreline, N.Y.U., Inst. Math. Sci., Div. EM Res., Research Report No. EM-46, July, 1952.

3. P. C. Clemmow, Radio propagation over a flat earth across a boundary separating two different media, Philos. Trans. Roy. Soc. London, vol. 246 (1953) pp. 1-55.

4. A. L. Cullen, The excitation of plane surface waves, Proceedings of the Institute of Electrical Engineers vol. 101 (1954) pp. 225.

5. L. B. Felsen, Field solutions for a class of corrugated wedges and cone surfaces, Polytech. Inst. of Brooklyn, Memo No. 32, July, 1957.

6. H. M. Barlow and A. L. Cullen, Surface waves, Proceedings of the Institute of Electrical Engineers vol. 100 (1953) p. 329.

7. J. Kane and S. N. Karp, An accurate boundary condition to replace transition conditions at dielectric-dielectric interfaces, N.Y.U., Inst. Math. Sci., Div. EM Res., Research Report No. EM-153, May, 1960.

8. C. Müller, Grundprobleme der mathematischen Theorie elektromagnetischer schwingungen, Göttingen, Springer-Verlag, 1927.

UNIVERSITY OF RHODE ISLAND 